\title{
The Care of Asthma Patients in Communities with Limited Resources
}

This article was published in the following Dove Press journal:

Research and Reports in Tropical Medicine

\author{
Basim A Dubaybo (ID \\ Faculty Affairs and Professional \\ Development, Wayne State University \\ School of Medicine, Detroit, MI, USA
}

\begin{abstract}
Asthma care has undergone significant changes over the past several years. Our understanding of the pathophysiology, risk factors, diagnosis, treatment, follow-up and prognosis has changed as more investigations shed new light on all of these aspects. This complexity has resulted in global guidelines aimed at improving the management of asthma patients. Among the multitude of guidelines, those published by the Global Initiative for Asthma (GINA) have achieved widespread and global acceptance. These guidelines cover the spectrum of factors that impact asthma care and include specific recommendations that treating physicians should follow to provide optimal patient care. In this review of asthma care in adult patients, we will outline some of the recommendations issued by GINA and discuss the challenges and limitations to the implementation of these guidelines in communities with limited resources. We will also propose recommendations to overcome these limitations in adult patients.
\end{abstract}

Keywords: asthma, limited resources, GINA

\section{Characteristics of Asthma in Communities with Limited Resources}

Several investigators have reported that the prevalence of asthma in communities with limited resources is at least as that in rich countries, but with higher severity. ${ }^{1,2}$ Furthermore, asthma control is less robust due to several factors including poor access to clinical services and medications, weak infrastructures and availability of pulmonary function testing sites, lack of asthma education, and several social and cultural factors resulting in poor compliance. ${ }^{3-5}$

\section{The GINA Guidelines: Historical Perspective}

In 1993, the Global Initiative for Asthma (GINA) was established by the World Health Organization and the National Heart Lung and Blood Institute. ${ }^{6}$ Since then, there have been multiple revisions of the guidelines, the last one being published in $2020 .^{7}$ The objectives of this initiative were to increase awareness about asthma among regulators, health authorities, providers, patients, and communities. The ultimate goal was to improve asthma prevention and management through a coordinated global effort. ${ }^{6,7}$ The multifaceted plan that GINA employed included preparation of scientific reports on asthma, encouragement of dissemination of this information, advocating for effective implementation of the recommendations, and promotion of global collaboration in the area of asthma research. ${ }^{8}$
Correspondence: Basim A Dubaybo Vice Dean for Faculty Affairs and Professional Development, Wayne State University School of Medicine, 540 E. Canfield Street, 1213 Scott Hall, Detroit, MI

Tel + I 3|3-577-1238

Email bdubaybo@med.wayne.edu
Research and Reports in Tropical Medicine 2021:12 33-38 


\section{Application of GINA Guidelines in Communities with Limited \\ Resources}

The latest published GINA guidelines ${ }^{7}$ include the following eight sections:

1. Definition, description, and diagnosis of asthma

2. Assessment of asthma

3. Treating asthma to control symptoms and minimize risk

4. Management of worsening asthma and exacerbations

5. Diagnosis and initial treatment of adults with features of asthma, COPD or both ("asthma-COPD overlap")

6. Diagnosis and management of asthma in children 5 years and younger

7. Primary prevention of asthma

8. Implementing asthma management strategies into health systems.

In this review, we have examined each of these sections and how it can be addressed in communities with limited resources. We have consolidated some of these sections (sections 3, 4 and 5 as well as sections 6,7 , and 8) to avoid redundancy.

\section{Optimizing the Diagnosis of Asthma Background}

The first area that GINA addressed is optimizing the diagnosis of asthma. Asthma had already been redefined as a heterogeneous disease, usually characterized by chronic airway inflammation, with symptoms of varying intensity including wheezing, shortness of breath, chest tightness and cough with variable expiratory airflow limitation. ${ }^{9}$ Over time, airflow limitation may later become persistent in the latter stages of the disease.

To provide an accurate and optimal diagnosis, therefore, providers and patients need to have a thorough understanding of the disease and other conditions that mimic it. Ensuring that there is adequate education for the providers who in turn provide adequate education to the patients is necessary to improve care.

\section{Challenges Faced in Low Resource}

\section{Communities}

Numerous studies have demonstrated that physicians taking care of asthma patients may not have a very detailed understanding of the GINA diagnostic guidelines. Alshafa compared internal medicine and family medicine doctors and their knowledge of GINA guidelines in a Saudi Arabian Hospital. Whereas the authors found no significant difference in knowledge between the two groups, the average knowledge of the diagnostic criteria was only around $50 \%{ }^{10}$ Similarly, a study from 2 teaching hospitals in Nigeria evaluating the asthma management practices of pulmonologists, internal medicine, family medicine and training physicians. There was general poor understanding of the GINA guidelines with average total knowledge score rated as poor $(37.2 \%){ }^{11}$ We also demonstrated a similar finding in Syria. ${ }^{12}$ In a recent review, it has been reported that, among doctors working in primary care health services, the precision of the diagnosis of asthma is far from ideal, varying from 54\% underdiagnosis to $34 \%$ over-diagnosis. ${ }^{13}$

Lack of familiarity with these guidelines by physicians translates into poor patient understanding of the elements of the disease and overall poor compliance and poor outcome. In a recent review of asthma care in Latin American nations, Sanchez-Borges found that there are several factors that influence patient outcome, poor patient education by practitioners being one of them. ${ }^{2}$

\section{Recommendations}

To improve physician and patient understating of the disease, there should be structured educational programs to disseminate knowledge. Different localities may have difference strategies. Some specific recommendations that have been successful include one-on-one teaching, continuing medical education courses, embedding specialists in teaching hospitals, distribution of audiovisual and printed material and others. Various studies have shown that these strategies improve knowledge and patient outcome. ${ }^{11,12}$

\section{Optimizing Asthma Assessment and Follow-Up Background}

Another aspect of the GINA guidelines to improve asthma care is the implementation of effective processes to assess patients in an ongoing manner. ${ }^{7,8}$ The guidelines not only intend to optimize symptom control but also evaluate the risks of severe adverse outcomes. Ultimately, the purpose is to evaluate symptom control, response to therapy, quality of life, and potential for adverse reactions and complications. 
Optimal assessment of Asthma symptom control requires age and gender appropriate evaluation tools that are individualized and inquire about the frequency or severity of symptoms, and achievement of established goals including school attendance, absence from work or performance of activities of daily living. ${ }^{14,15}$ In addition, optimal assessment includes identification of the potential for adverse asthma outcomes such as acute exacerbations, persistent airflow limitation, and side-effects of treatment strategies. Of particular interest is the use of high medication doses and poor inhaler techniques. GINA guidelines indicate that impaired pulmonary function tests are the most useful indicators of future risk and should be recorded at diagnosis, 3-6 months after starting treatment, and periodically thereafter. ${ }^{6-9}$

\section{Challenges Faced in Low Resource Communities}

An effective assessment strategy requires the availability of trained providers, comprehensive assessment tools, pulmonary function testing sites and equipment, access to these facilities, patient understanding and willingness to accept findings and recommendations. We and others have identified a number of barriers that negatively impact the ability of patients residing communities with limited resources to get accurate and timely assessments. ${ }^{12,16}$ In a comprehensive review, Majellano outlined these barriers in great detail. ${ }^{16}$ The author indicated that these barriers may be related to several factors including limited number of available and well-trained physicians, poor communications, poor understanding, and social factors that prevent compliance.

Providers can benefit from the availability of simple screening tools that address these issues. The assessment tools can be tailored to meet the needs of individual patients such as their age and gender. When asthma control is assessed effectively, providers can ascertain whether the treatment is effective, and to what degree the symptoms are related to severe asthma, uncontrolled asthma, poor medication compliance, or incorrect inhaler technique. Several assessment tools exist. ${ }^{6-9}$ Pulmonary function tests in a controlled laboratory setting remain the most reliable and accurate assessment tool. ${ }^{17}$ However, it is expensive and not widely available in a timely manner to all patients in under-resourced communities. Where not available, providers can use simple portable spirometry or peak flow meters. Although these have limited applications, they can provide very valuable information. Other tools have also been proposed and described in a comprehensive review by Al-Zahrani. ${ }^{18}$ These include

- Asthma Control Test

- Childhood Asthma Control Test

- Responsiveness

- Asthma Control Questionnaire

- Asthma Therapy Assessment Questionnaire

- Lara Asthma Symptom Scale.

\section{Recommendations}

To overcome barriers to effective assessment of severity, control and risk for exacerbations, emphasis in low resource communities should focus on three parameters. The first specific recommendation is increasing the pool of providers who are able to provide these assessments. This is accomplished by using primary care providers as well as mid-level providers and trainees in the assessment process. ${ }^{12}$ The second parameter is improving education of both providers and patients. This may be accomplished by a variety of strategies ${ }^{19}$ including participating in global efforts such as GINA. ${ }^{6-9}$ And finally, there is a need to increase the availability of assessment tools. ${ }^{16-18}$ These are also available through global networks and are listed above. ${ }^{7}$ Overcoming these barriers allows patients to enroll in structured programs of follow-up and management.

To ensure that that assessment tools are available to patients, it is important to these patients have access to healthcare facilities. Since this is a challenge in communities with limited resources, investigators have proposed strategies to improve access. Among these strategies, those proposed in 2018 by the Global Asthma Network, are among the most comprehensive ${ }^{20}$ and include steps that can be taken by patients, providers, governments, and international agencies to assist in this regard.

\section{Control of Asthma Symptoms and Risk of Exacerbations \\ Background}

In order to improve control of asthma symptoms and reduce the risk of exacerbations, GINA proposes an approach that includes population-based decisions as well as patient-level individualized decisions. ${ }^{6-9}$ Both factors are influenced by local regulations and access. The population-level approach includes national 
guidelines and health maintenance organizations ${ }^{8}$ and is generally based on group mean data for symptoms, lung function and exacerbations, as well as safety, availability and overall cost. Individualized patient decisions require the clinicians understand patient characteristics that may influence the rate of exacerbations and response to therapy. These include such factors as age, smoking, sputum eosinophils, allergies, comorbidities, health literacy, family history, and others. Patient preferences and skills are paramount especially as it pertains to the choice of inhalers, ability to master inhaler techniques, compliance, and cost. ${ }^{13-15}$

In practice, as is articulated in GINA guidelines, improving control of asthma symptoms requires a multifaceted approach to therapy. Providers need to identify and manage risk factors and encourage smoking cessation weight reduction, and other non-pharmacological interventions such as physical activity, breathing exercises and avoidance of allergens. Finally, a step-up approach to therapy is indicated. ${ }^{8}$

It is important to remember that many patients, particularly older individuals with smoking history may have features of both asthma and COPD. Some patients may, in fact, have asthma-COPD overlap syndrome (ACOS). This creates therapeutic challenges especially when patients are managed by non-specialists. ${ }^{21}$ In asthma, inhaled corticosteroids (ICS) are the cornerstone of treatment and there are strong recommendations against use of long-acting betaagonists (LABA) as monotherapy, while, for COPD, LABA monotherapy is recommended. Therefore, it is important to identify this group and offer them individualized care.

\section{Challenges Faced in Low Resource Communities}

Limited access to care remains an important barrier to effective care and prevention of exacerbations. This includes limited number of primary care centers, limited number of centers than can adequately evaluate response to therapy, and limited availability of specialists. It also includes limited availability of medical educators who help both providers and patients adhere to sound clinical practices. In addition, limited health literacy has significant untoward effects by impairing adherence to asthma treatment plan and medication administration.

\section{Recommendations}

The main recommendations in this area include emphasis on improving health literacy among patients and providers. Patients need to learn useful details about the disease, the importance of compliance with medications and action plan, modifiable risk factors and signs of serious life-threatening flare-ups. To improve health literacy and subsequent patientcentered outcomes, tailored communication regarding the risks and benefits of tests and treatments such as educational material as the GINA patient Guide. This publication is available widely.

Providers need to be comfortable using controller agents, specifically inhaled corticosteroids, to improve control and decrease the risk of serious complications and death. Health departments need to accentuate provider education programs.

Another main factor that is needed to improve control is increased access to healthcare for asthma patients. This entails training primary care doctors and providing alternative methods of communicating with patients as described above.

\section{Implementing Asthma Management Strategies into Health Systems Background}

To improve asthma care, including the areas of preventive and pediatric care, national and local health authorities must adopt and implement evidence-based policies and procedures and ensure they are integrated into clinical practice. There are several steps that are needed to execute this strategy. ${ }^{6-9}$ These include:

- Development of national multidisciplinary working groups which include stakeholders.

- Assessment of current asthma care needs, considering local cultural and socioeconomic conditions.

- Selection of cost-effective goals.

- Identification of key recommendations for diagnosis and treatment that are appropriate for the current cultural conditions.

- Identification and developing strategies to confront barriers to implementation.

- An implementation framework and its component strategies.

- Develop a step-by-step implementation plan. 


\section{Challenges Faced in Low Resource Communities}

Many barriers to the implementation of national health strategies exist. ${ }^{6-9}$ Some relate to health care providers, including insufficient knowledge of asthma management recommendations, lack of agreement with recommendations, skepticism about the effectiveness of these recommendations, resistance to change, limited time and resources, and concerns about litigation and medicolegal issues. ${ }^{8}$ Other barriers relate to patients. These are mainly cultural and economic barriers and include low health literacy, insufficient understanding of asthma and its management, attitudes, beliefs, preferences, anxieties, and misconceptions. ${ }^{8}$

\section{Recommendations}

To overcome these barriers, healthcare providers need to be brought on board. This includes an educational campaign that includes specialists as well as other providers. Here, communities with limited resources may benefit from assistance from national and global organizations who are ready to assist. To overcome patient-specific barriers, a comprehensive patient education program is required. This includes commitment from authorities and well as support from global organizations. Two specific interventions are needed. First, there is a need for increased funding of public health programs at all levels, including those targeting asthma management. In addition, outcome measures of efficacy are needed to ensure efficient utilization of resources.

\section{Summary}

Global guidelines to improve the care of asthma patients have been developed and found to be effective in improving patient care. Significant barriers to their implementation exist in communities with limited resources. These barriers are related to two main issues, lack of education and lack of access. Both issues can be overcome through coordinated efforts between healthcare providers, local authorities, and global organizations that can complement local resources.

\section{Disclosure}

The author reports no conflicts of interest in this work.

\section{References}

1. Kuti BP, Omole K, Kuti D, et al. Factors associated with childhood asthma control in a resource-poor center. J Family Med Prim Care. 2017;6(2):222-230. doi:10.4103/jfmpc.jfmpc_271_16

2. Sánchez-Borges M, Capriles-Hulett A, Caballero-Fonseca F. Asthma care in resource-poor settings. World Allergy Organ J. 2011;4 (4):68-72. doi:10.1097/WOX.0b013e318213598d

3. Al-Muhsen S, Dulgom S, Assiri Z, et al. Poor asthma education and medication compliance are associated with increased emergency department visits by asthmatic children. Ann Thorac Med. 2015;10 (2):123-131. doi:10.4103/1817-1737.150735

4. Bryant-Stephens T, Reed-Wells S, Canales M, et al. Home visits are needed to address asthma health disparities in adults. $J$ Allergy Clin Immunol. 2016;138(6):1526-1530. doi:10.1016/j.jaci.2016.10.006

5. McQuaid EL. Barriers to medication adherence in asthma: the importance of culture and context. Ann Allergy Asthma Immunol. 2018;121 (1):37-42. doi:10.1016/j.anai.2018.03.024

6. Boulet LP, Reddel HK, Bateman E, Pedersen S, FitzGerald JM, O'Byrne PM. The Global Initiative for Asthma (GINA): 25 years later. Eur Respir J. 2019;54(2):1900598. PMID: 31273040. doi:10.1183/13993003.00598-2019

7. Global Initiative for Asthma. Pocket guide for asthma management and prevention; 2020. Available from: https://ginasthma.org/wpcontent/uploads/2020/04/Main-pocket-guide_2020_04_03-final-wms. pdf. Accessed January 1, 2021.

8. Reddel HK, Bateman ED, Becker A, et al. A summary of the new GINA strategy: a roadmap to asthma control. Eur Respir J. 2015;46 (3):579-582. doi:10.1183/13993003.00853-2015

9. Reddel H, Levy M. The GINA asthma strategy report: what's new for primary care? Prim Care Resp Med. 2015;25(1):15050. doi:10.1038/ npjpcrm.2015.50

10. Alshafa SM, Alshehri NM. Assessment of family and internal medicine physicians knowledge and practice of bronchial asthma at Riyadh city. J Family Med Prim Care. 2020;9(8):4358-4362. doi:10.4103/jfmpc.jfmpc_1233_19

11. Umoh V, Akpan E, Aluka A, Alasia D, Ekott J. An assessment of physicians' knowledge of the GINA guidelines. Eur Respir J. 2011;38:p2851.

12. Mohammad Y, Shaaban R, Salman HA, Shabraq BN, Dubaybo B. Improving the quality of hospital care provided for asthma out-patients in a country in turmoil: a report from Syria. $J$ Thorac Dis. 2019;11(3):1047-1055. PMID: 31019794; PMCID: PMC6462698. doi:10.21037/jtd.2019.02.76

13. Jose BP, Camargos PA, Cruz Filho AA, Correa Rde A. Diagnostic accuracy of respiratory diseases in primary health units. Rev Assoc Med Bras. 2014;60(6):599-612. doi:10.1590/1806-9282.60.06.021

14. Dinakar C, Chipps BE. Clinical tools to assess asthma control in children. Pediatrics. 2017;139(1):e20163438. doi:10.1542/ peds.2016-3438

15. Horne R, Price D, Cleland J, et al. Can asthma control be improved by understanding the patient's perspective? BMC Pulm Med. 2007;7 (1):8. doi:10.1186/1471-2466-7-8

16. Majellano EC, Clark VL, Winter NA, Gibson PG, McDonald VM. Approaches to the assessment of severe asthma: barriers and strategies. J Asthma Allergy. 2019;12:235-251. doi:10.2147/JAA. S178927

17. Culver BH, Graham BL, Coates AL, et al. ATS committee on proficiency standards for pulmonary function laboratories. recommendations for a standardized pulmonary function report. an official American thoracic society technical statement. Am J Respir Crit Care Med. 2017;196(11):1463-1472. doi:10.1164/rccm.2017101981ST PMID: 29192835. 
18. Alzahrani YA, Becker EA. Asthma control assessment tools. Respiratory Care. 2016;61(1):106-116. doi:10.4187/respcare.04341

19. Larsson K, Kankaanranta H, Janson C, et al. Bringing asthma care into the twenty-first century. NPJ Prim Care Respir Med. 2020;25:30. doi:10.1038/s41533-020-0182-2

20. Ellwood P, Ellwood E, Rutter C, et al.; on behalf of the GAN Phase I Study Group. Global asthma network Phase I surveillance: geographical coverage and response rates. J Clin Med. 2020;9(11):3688. doi: $10.3390 / \mathrm{jcm} 9113688$
21. Tho NV, Park HY, Nakano Y. Asthma-COPD overlap syndrome (ACOS): a diagnostic challenge. Respirology. 2016;21(3):410-418. PMID: 26450153. doi:10.1111/resp.12653

\section{Publish your work in this journal}

Research and Reports in Tropical Medicine is an international, peerreviewed, open access journal publishing original research, case reports, editorials, reviews and commentaries on all areas of tropical medicine, including: Diseases and medicine in tropical regions; Entomology; Epidemiology; Health economics issues; Infectious disease; Laboratory science and new technology in tropical medicine;
Parasitology; Public health medicine/health care policy in tropical regions; and Microbiology. The manuscript management system is completely online and includes a very quick and fair peer-review system. Visit http://www.dovepress.com/testimonials.php to read real quotes from published authors. 